\title{
Probabilistic Map Building FOR AIRCRAFT-TRACKING RADARS*
}

\author{
João P. Hespanha \\ hespanha@usc.edu \\ Hüseyin H. Kızılocak \\ Yusuf S. Ateşkan \\ kiziloca@usc.edu \\ ateskan@usc.edu \\ Dept. of Electrical Engineering-Systems, University of Southern California, Los Angeles, CA
}

\begin{abstract}
In this paper we develop a formalism to assess the risk of missions that consist of flying an aircraft over a region infested by enemy radars and co-located Surfaceto-Air Missile (SAM) launchers. This is accomplished by building several probabilistic maps that will allow us to compute the probability of success for the missions.
\end{abstract}

\section{Introduction}

The future of air-operations is evolving towards a scenario where the battlefield is populated with many unmanned air-vehicles gifted with a large number of cheap sensors whose primary mission is to gather information about enemy assets such as radars with co-located SAM launchers and possibly execute simple missions in an autonomous fashion. In this scenario, the fusion of information coming from a multitude of sensors into forms suitable for mission planning is a major challenge because the data gathered is likely to be noisy. This paper addresses precisely this problem. In particular, we develop a probabilistic formalism to process the information coming from aircraft sensors capable of detecting electromagnetic radiation originating from radars, making it suitable for the planning of air-operations. The need for a probabilistic framework stems from the fact that the sensors producing the measurements are not perfect and are susceptible to provide erroneous information. Because of this, inferences made from this data can never be assumed 100\% correct.

A key concept used in this paper is that of a probabilistic map. In usual maps of a region, one finds markings that represent locations of objects (e.g., radars) within the region. By placing one of these markings in a location on the map, one is expressing the fact that the object represented can be found at that particular location with probability one. However, when the information used to build a map (or more precisely to

${ }^{*}$ This research was supported by the Defense Advanced Research Projects Agency and the Office of Naval Research.

In Proc. of the 2001 American Control Conference. locate interesting objects in it) is not perfect, it is not possible to mark a specific position on the map as the location of the object. In this case, the best one can do is to superimpose the probability density of the location of the object in question on the whole map. It is this probability density that we call the probabilistic map for the object. From a formal point of view, probabilistic maps are actually probability densities of the position of objects, conditioned to the available sensor measurements. Note that these probabilistic maps do not represent the geography of a region, but are often related to it, e.g., the probabilistic map of a radar will most likely exhibit zero probability over areas covered by a river because it is unlikely to find a radar over water.

The main difficulty in computing probabilistic maps is their size. When one is mapping multiple objects whose positions are not independent of each otherand therefore one needs to keep track of the joint probability density of all the objects - the probabilistic map (i.e., the joint probability density) easily becomes very large. This is because, in principle, the memory required to represent a joint probability density grows exponentially with the number of random variables. It should be noted that (conditional) independence of the positions of several radars is not expected in our problem because, e.g., the probability of all the radars being very close to each other is often much smaller (or at least different) than the probability of the radars being spread to cover a certain perimeter.

This paper contains two main contributions: First we show that, for the sensor models used here, the probabilistic map of a large number of radars can be represented by a function-called the aggregate measurement function - defined in a low-dimensional space (two-dimensional for the specific setup in this paper). We then show that it is possible to assess the risk of given missions in air-operations and even do minimumrisk path planning without ever explicitly computing the high-dimensional probabilistic map and working simply with the aggregate measurement function. The algorithms presented here were implemented in a simulator of military air-operations developed by Honeywell 
Technology Center and were used to guide aircraft in strike missions.

Probabilistic map building is becoming an important topic in the robotics literature. Especially for indoors robotics, map building is often combined with the problem of localizing the robot within a global coordinate system $[1,2,3,4,5,6]$. This is required since the measurements used to build the map are a function of the position of the robot, which is hard to determine accurately due to accumulated odometry errors. In outdoors robotics, the localization problem can be mitigated through the use of GPS. Occupancy grid mapping is currently the most widely used class of mapping algorithms for mobile robots (cf. , e.g., [3, 7]). Occupancy maps simply provide the probability of each individual cell in the grid being occupied by an object. This information is not sufficient to compute the probability of a particular configuration of objects, unless it is assumed that the existence of objects in different cells correspond to independent events. As mentioned above, it is difficult to justify this type of independence assumption for the problem at hand.

This paper is organized as follows. In Section 2, we formally define the radar probabilistic map in terms of a joint conditional distribution and then proceed to show how this map can be computed from data acquired by a noisy sensor. The key result in this section is that, although a probabilistic map is a function defined in a high-dimensional space, it can be represented compactly by another function that takes values in a space with the same dimension as the region where the radars are located. In Section 3, we define the radar occupancy map and show how this map is related to the radar probabilistic map. The main result of this section is an algorithm to efficiently compute the occupancy map directly from the compact representation of the radar probabilistic map. In Section 4, we show how to compute a danger or kill-map that measures the danger of flying over a certain point in the mapped region. This map is then used to compute the probability of success of a particular flight mission. This probability (or more precisely the logarithm of it) is linear on the values of a simple transformation of the kill-map and is therefore computationally amenable to do minimum-risk path planning. The algorithms presented here were implemented in Honeywell's simulator of military air-operations and the results are shown in Section 5. Finally, Section 6 contains some concluding remarks and directions for future research. The reader is referred to [8] for some derivations omitted here due to space limitations.

\section{Probabilistic maps for aircraft-tracking radars}

Suppose that there exist $n$ radars in a region $\mathcal{R} \subset \mathbb{R}^{2}$, each radar capable of tracking any aircraft that enters its range. For now we assume that the positions of these radars are fixed but not accurately known. For simplicity we take $\mathcal{R}$ to be a finite collection of points, each representing a cells in $\mathbb{R}^{2}$. We model the position of each radar as a random variable ${ }^{1}$ taking values in $\mathcal{R}$. We denote by $\mathbf{r}:=\left\{\mathbf{r}_{1}, \mathbf{r}_{2}, \ldots, \mathbf{r}_{n}\right\} \in \mathcal{R}^{n}$ the positions of the $n$ radars, where $\mathbf{r}_{i} \in \mathcal{R}$ denotes the position of the $i$ th radar. We assume that an a priori joint probability distribution for the radars

$$
p_{0}(r):=\mathrm{P}\left(\mathbf{r}_{1}=r_{1}, \mathbf{r}_{2}=r_{2}, \ldots, \mathbf{r}_{n}=r_{n}\right),
$$

$r:=\left\{r_{1}, r_{2}, \ldots, r_{n}\right\} \in \mathcal{R}^{n}$ is known. This a priori probability distribution may reflect geographical information, the fact that radars will not be too close to each other, etc. We also assume the radars to be $a$ priori indistinguishable, meaning that for any permutation $j:=\{j(1), j(2), \ldots, j(n)\}$ of $\{1,2, \ldots, n\}$ we have

$$
p_{0}\left(\left\{r_{1}, r_{2}, \ldots, r_{n}\right\}\right)=p_{0}\left(\left\{r_{j(1)}, r_{j(2)}, \ldots, r_{j(n)}\right\}\right) .
$$

Our knowledge about the position of the radars improves with time through observations collected by the aircraft flying over $\mathcal{R}$. These observations are denoted by $\mathbf{y}_{k}, k \in \mathcal{K}:=\{1,2, \ldots, K\}$, and each $\mathbf{y}_{k}$ consists of a triple of the form

$$
\left\{\mathbf{t}_{k}, \mathbf{a}_{k}, \mathbf{s}_{k}\right\}
$$

where $\mathbf{t}_{k} \geq 0$ denotes the time at which the observation was made and $\mathbf{a}_{k} \in \mathcal{R}$ the position of the aircraft. The variable $\mathbf{s}_{k}$ can either have the value $\mathbf{s}_{k}=\emptyset$ if the aircraft was not being tracked at that time or some value $\mathbf{s}_{k} \in \mathcal{R}$ if it was being tracked from a radar positioned at $\mathbf{s}_{k}$. However, we assume that the sensors used to detect tracking radars are not perfect and therefore $\mathbf{s}_{k}=\emptyset$ simply means that the sensors were not able to detect a radar and one should consider that this may be a "false negative." Similarly, $\mathbf{s}_{k} \in \mathcal{R}$ may be caused by a "false positive," or there may be a radar close to $\mathbf{s}_{k}$ but not exactly at that location. The set of all possible observation triples is denoted by $\mathcal{Y}$.

Sensors of this type can be modeled by a stationary likelihood function of the form

$\ell_{n}(y, r):=\mathrm{P}\left(\mathbf{y}_{k}=y \mid \mathbf{r}=r\right), \quad k \in \mathcal{K}, r \in \mathcal{R}^{n}, y \in \mathcal{Y}$.

We refer to $\ell_{n}$ as the $n$-radar sensor likelihood function. Here, we assume that $\mathbf{y}_{k}$ is conditionally independent of any other measurements, given a particular configuration $\mathbf{r}=r$ for the radars. This implicitly assumes

\footnotetext{
${ }^{1}$ In this paper we denote random variables by bold symbols and their realizations by non-bold ones.
} 
that sensor errors occur independently of each other. The stationarity assumption could easily be lifted but we keep it here for simplicity of notation.

The goal of this section is to introduce the a posteriori probabilistic map for the radars defined by

$$
m_{r}(r, Y):=\mathrm{P}\left(\mathbf{r}=r \mid \mathbf{Y}_{K}=Y\right), \quad r \in \mathcal{R}^{n},
$$

where $\mathbf{Y}_{K}$ denotes the list $\left\{\mathbf{y}_{1}, \mathbf{y}_{2}, \ldots, \mathbf{y}_{K}\right\}$ of all sensor observations available. Since we will compute $m_{r}$ recursively, it will be convenient to build probabilistic maps with just a subset of the observations available. With a slight abuse of notation we will use the same symbol $m_{r}$ for the $k$-observations probabilistic map for the radars, $k \in \mathcal{K}$, defined by

$$
m_{r}\left(r, Y_{k}\right):=\mathrm{P}\left(\mathbf{r}=r \mid \mathbf{Y}_{k}=Y_{k}\right), \quad r \in \mathcal{R}^{n},
$$

where $\mathbf{Y}_{k}$ denotes a list $\left\{\mathbf{y}_{1}, \mathbf{y}_{2}, \ldots, \mathbf{y}_{k}\right\}$ with the first $k$ sensor observations. In the sequel we denote by $\mathcal{Y}^{*}$ the set of all possible lists of measurements with any length.

\subsection{Single-radar sensor likelihood functions}

In practice, the precise shape of the sensor likelihood function must be estimated experimentally. The following lemma gives a simple parametric model for a singleradar (i.e., $n=1$ ) sensor likelihood function that could be "best-fitted" to experimental data. This model is defined by the following parameters:

- $\rho_{\text {radar }}>0$ : range over which the radar is capable of tracking aircraft.

- $p_{\text {track }} \in[0,1]$ : probability that the radar will track an aircraft within its range (in general, could be a function of the radar position and the enemy's intention).

- $\rho_{\text {sensor }}>0$ : range over which the sensor can detect a radar tracking the aircraft.

- $p_{\text {true }}$ : probability that the sensor will detect the radar, given that it is being tracked. We assume that within the range defined by $\rho_{\text {sensor }}$ this probability is approximately constant.

- $e_{\mathrm{loc}}$ : maximum localization error for the position of the radar when the sensor correctly detects that the aircraft is being tracked. The actual localization error is assumed uniform within the range specified by $e_{\text {loc }}$. We also assume here that $e_{\text {loc }}$ increases linearly with the distance between the aircraft and the radar, i.e., $e_{\text {loc }}(\|r-a\|)=e_{\min } \frac{\sqrt{\|r-a\|^{2}+h^{2}}}{h}$, where $h$ denotes the aircraft altitude. This would be consistent with a fixed uniform distribution for the error in units of angle.

- $p_{\text {false }}$ : probability that the sensor will detect a radar tracking the aircraft when this is not happening. We assume here that the distribution for the position of the radar "detected" is uniform over the region $\{\bar{s} \in$ $\left.\mathcal{R}:\|a-\bar{s}\|<\rho_{\text {sensor }}\right\}$. For any useful sensor we should always have $p_{\text {false }}<p_{\text {true }}$.
Lemma 1 [8] The single-radar sensor likelihood function consistent with the parameters above is given by

$$
\ell_{1}(\{t, a, s\}, r)= \begin{cases}\frac{p_{\text {false }}}{A(a)} & \|a-s\| \leq \rho_{\text {sensor }} \\ 0 & \text { otherwise }\end{cases}
$$

when $s \in \mathcal{R}$ and $\|r-a\|>\rho_{\text {radar }}$, by

$$
\begin{aligned}
\ell_{1}(\{t, a, s\}, r)= & \begin{cases}\frac{p_{\text {true }} p_{\text {track }}}{B(\|r-a\|)} & \|s-r\| \leq e_{\text {loc }}(\|r-a\|) \\
0 & \text { otherwise }\end{cases} \\
& + \begin{cases}\frac{p_{\text {false }}\left(1-p_{\text {track }}\right)}{A(a)} & \|a-s\| \leq \rho_{\text {sensor }} \\
0 & \text { otherwise }\end{cases}
\end{aligned}
$$

when $s \in \mathcal{R}$ and $\|r-a\| \leq \rho_{\text {radar }}$ and by

$\ell_{1}(\{t, a, \emptyset\}, r)=$

$\begin{cases}1-p_{\text {true }} p_{\text {track }}-p_{\text {false }}\left(1-p_{\text {track }}\right) & \|r-a\| \leq \rho_{\text {sensor }} \\ 1-p_{\text {false }} & \text { otherwise }\end{cases}$

when $s=\emptyset$. Here, $A(a)$ and $B(b)$ denote the areas of the regions $\left\{\bar{s} \in \mathcal{R}:\|a-\bar{s}\| \leq \rho_{\text {sensor }}\right\}$ and $\left\{\bar{s} \in \mathcal{R}:\|\bar{s}-r\| \leq e_{\text {loc }}(b)\right\}$ respectively.

A typical single-radar sensor likelihood function is plotted in Figure 1. The parameters used for these plots were: $\rho_{\text {radar }}=3, p_{\text {track }}=.9, \rho_{\text {sensor }}=4, p_{\text {true }}=.95$, $h=2, e_{\min }=1$, and $p_{\text {false }}=.05$.
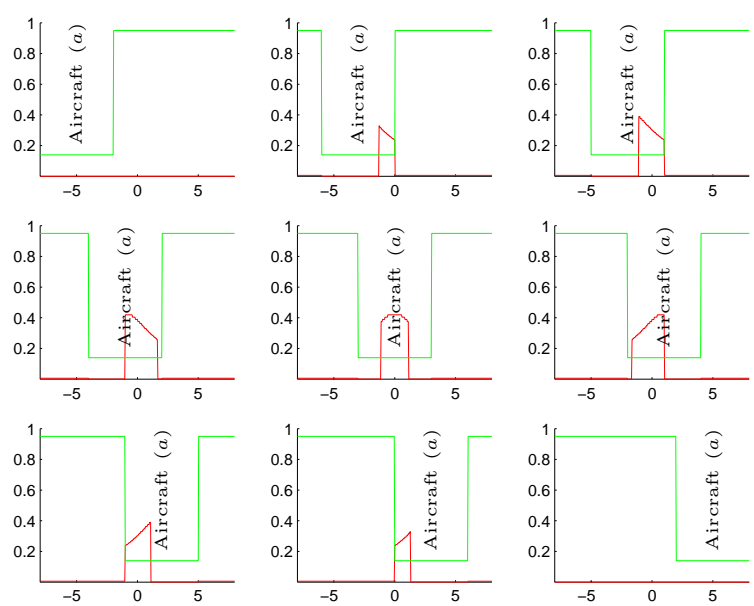

Figure 1: Typical single-radar sensor likelihood function. The plots represent $\ell_{1}(\{t, a, \emptyset\}, r)$ (light line) and $\ell_{1}(\{t, a, 0\}, r)$ (dark line) versus the radar position $r$ for several positions $a$ of the aircraft.

\subsection{Multiple-radar sensor likelihood functions}

The likelihood function for multiple radars given in the next lemma is based on the following assumptions:

1. From the sensor point of view, the radars are indistinguishable from each other. 
2. The aircraft can only be within the range of at most one radar. This is true, for example, when the distance between any two radars is larger than their range $\rho_{\text {radar }}{ }^{2}$.

Lemma 2 [8] The n-radar sensor likelihood function is given by

$$
\ell_{n}(y, r)=c \prod_{i=1}^{n} \ell_{1}\left(y, r_{i}\right), \quad y \in \mathcal{Y}, r \in \mathcal{R}^{n}
$$

where $c$ is a normalizing constant and $\ell_{1}$ is the singleradar likelihood function.

\subsection{Probabilistic map for the radars}

Pick now some $k \in \mathcal{K}$ and a list $Y_{k}:=\left\{y_{1}, y_{2}, \ldots, y_{k}\right\}$ of $k$ measurements. Using Bayes rule we conclude that,

$$
m_{r}\left(r, Y_{k}\right)=c_{k} \ell_{n}\left(y_{k}, r\right) m_{r}\left(r, Y_{k-1}\right)
$$

where $Y_{k}:=\left\{Y_{k-1}, y_{k}\right\}, r \in \mathcal{R}^{n}$ and $c_{k}>0$ is a constant chosen so that $\sum_{r \in \mathcal{R}} m_{r}\left(r, Y_{k}\right)=1$. When the $a$ priori probabilistic map precludes configurations where the distance between two radars is smaller than $\rho_{\text {radar }}$, the likelihood function $\ell_{n}$ given in Section 2.2 is valid and iterating (3) from 1 through $k$, we get

$$
m_{r}\left(r, Y_{k}\right)=\hat{c}_{k}\left(\prod_{i=1}^{n} f\left(r_{i}, Y_{k}\right)\right) p_{0}(r)
$$

where $r:=\left\{\mathbf{r}_{1}, \mathbf{r}_{2}, \ldots, \mathbf{r}_{n}\right\} \in \mathcal{R}^{n}, \hat{c}_{k}$ is a normalizing constant and

$$
f\left(x, Y_{k}\right):=\prod_{j \in \mathcal{K}} \ell_{1}\left(y_{j}, x\right), x \in \mathcal{R} .
$$

The function $f\left(\cdot, Y_{k}\right)$ - called here the aggregate measurement function - encapsulates the information contained in all the measurement. This is important since for any region of reasonable size, the posterior probabilistic map $m_{r}\left(\cdot, Y_{k}\right)$ cannot be represented exhaustively because such representation would require a number of values of the order of $|\mathcal{R}|^{n}$, where $|\mathcal{R}|$ is the number of points in $\mathcal{R}$. However, using (4) one can represent $m_{r}\left(\cdot, Y_{k}\right)$ using a single function $f\left(\cdot, Y_{k}\right)$, whose exhaustive representation only requires $|\mathcal{R}|$ values.

\section{Occupancy maps for aircraft-tracking radars}

In this section we introduce the a posteriori occupancy maps for the radars defined by

$$
m_{o}(x, Y):=\mathrm{P}\left(\exists i: r_{i}=x \mid \mathbf{Y}_{k}=Y\right), \quad x \in \mathcal{R},
$$

\footnotetext{
${ }^{2}$ In case some radars are placed very close to each other, we can just regard these radars as a single air-defense unit.
}

where $Y \in \mathcal{Y}^{*}$ denotes a list of $k$ observations. From the definition of the probabilistic map $m_{r}(\cdot, Y)$, we conclude that

$$
m_{o}(x, Y)=\sum_{\left\{r \in \mathcal{R}^{n}: \exists i: r_{i}=x\right\}} m_{r}(r, Y) .
$$

In this paper we consider a priori joint probability maps of the form

$$
\begin{aligned}
& p_{0}\left(\left\{r_{1}, r_{2}, \ldots, r_{n}\right\}\right):= \\
& \begin{cases}0 & \exists i \neq j:\left\|r_{i}-r_{j}\right\| \leq d_{\min } \\
\bar{c} \prod_{i=1}^{n} g_{0}\left(r_{i}\right) & \text { otherwise }\end{cases}
\end{aligned}
$$

where $\left\{r_{1}, r_{2}, \ldots, r_{n}\right\} \in \mathcal{R}^{n}, \bar{c}$ is a normalizing constant, and $g_{0}(\cdot)$ reflects geographical (or other) information making some cells in $\mathcal{R}$ more likely than others to contain radars. This map also encodes the facts that there is a minimum distance $d_{\text {min }}$ between any two of the radars and the radars are indistinguishable from each other.

Let us fix some $Y \in \mathcal{Y}^{*}$ and suppose that there exists a partition $\mathcal{R}_{0}, \mathcal{R}_{1}, \mathcal{R}_{2}, \ldots, \mathcal{R}_{m}$ of $\mathcal{R}$ such that all points in each $\mathcal{R}_{i}, i \in\{1,2, \ldots, m\}$, are away from each other by no more than $d_{\min }$, all the $\mathcal{R}_{i}, i \in\{1,2, \ldots, m\}$, are away from each other by more than $d_{\text {min }}$, the values of $f(z, Y)$ are sufficiently uniform over $\mathcal{R}_{0}$ so that, for any $\overline{\mathcal{R}} \subset \mathcal{R}_{0}$ with small area compared to $\mathcal{R}_{0}$,

$$
\sum_{z \in \mathcal{R}_{0} \backslash \overline{\mathcal{R}}} f(z, Y) g_{0}(z) \approx \sum_{z \in \mathcal{R}_{0}} f(z, Y) g_{0}(z) .
$$

For each $x \in \mathcal{R}$, let us denote by $\sigma:=\left\{\sigma_{1}, \sigma_{2}, \ldots, \sigma_{k}\right\}$ a combination of $k$ elements taken from the set $\{i: x \notin$ $\left.\mathcal{R}_{i}\right\} \subset\{1,2, \ldots, m\}$ and by $\mathcal{S}^{(k)}[x]$ the set of all such combinations. Then we have the following lemma:

Lemma 3 [8] The a posteriori occupancy map for the radars is given by

$$
\begin{aligned}
& m_{o}(x, Y) \approx \bar{c} f(x, Y) g_{0}(x)\left(n f_{0}(Y)^{n-1}+\right. \\
& \left.\sum_{k=1}^{n-1} \frac{n ! f_{0}(Y)^{n-k-1}}{(n-1-k) !} \sum_{\sigma \in \mathcal{S}^{(k)}[x]} f_{\sigma_{1}}(Y) f_{\sigma_{2}}(Y) \ldots f_{\sigma_{k}}(Y)\right),
\end{aligned}
$$

where $Y \in \mathcal{Y}^{*}$ denotes a list of $k$ observations $f_{i}(Y):=$ $\sum_{r \in \mathcal{R}_{i}} f(r, Y) g_{0}(r), i \in\{0,1, \ldots, m\}$, and $\bar{c}$ is a normalizing constant.

Lemma 3 allows us to compute the a posteriori occupancy map $m_{o}(\cdot, Y)$ directly from the aggregate measurement function $f(\cdot, Y)$, without explicitly computing the a posteriori probabilistic map $m_{r}(\cdot, Y)$. 


\section{Mission risk assessment}

In this section we introduce the probability of success of a particular flight mission using the a posteriori occupancy map defined in Section 3. To this effect, let us start by computing the a posteriori kill map defined by

$$
m_{\text {kill }}(a, Y):=\mathrm{P}\left(\mathfrak{K} \mid \mathbf{Y}_{k}=Y, \mathbf{a}_{k}=a\right)
$$

were $Y$ denotes a list of $k$ observations, $\mathfrak{K}$ the event that an aircraft positioned at $a$ will be tracked by a radar, shot at by a SAM co-located with the radar, and destroyed as a result. The kill map can be computed in terms of the previously defined parameters $\rho_{\text {radar }}$ and $p_{\text {track }}$, together with the probability $p_{\text {kill }} \in[0,1]$ that a missile will destroy an aircraft that is being tracked (in general, $p_{\text {kill }} \in[0,1]$ could be a function of the aircraft position and the enemy's intention). When the aircraft can only be within the range of at most one radar,

$$
m_{\text {kill }}(a, Y)=p_{\text {kill }} p_{\text {track }} \sum_{\|x-a\| \leq \rho_{\text {radar }}} m_{o}(x, Y)
$$

Suppose now that we are interested in having an aircraft flying a mission whose trajectory is defined by $m$ way points $\left\{x_{1}, x_{2}, \ldots, x_{m}\right\}$. The conditional a posteriori probability $p_{\text {survive }}$ that an aircraft will survive this mission, given a list of past measurement $\mathbf{Y}_{k}=Y \in \mathcal{Y}^{*}$, is given by

$$
p_{\text {survive }}=\prod_{j=1}^{m}\left(1-m_{\mathrm{kill}}\left(x_{j}, Y\right)\right)
$$

where $m_{\text {kill }}(x, Y)$ can be obtained from (6). It is often convenient to express this as a summation, which can be achieved taking the logarithm of $p_{\text {survive }}$ :

$$
\log \left(p_{\text {survive }}\right)=\sum_{j=1}^{m} \log _{\text {live }}\left(x_{j}, Y\right),
$$

where $\log _{\text {live }}(x, Y):=\log \left(1-m_{\text {kill }}(x, Y)\right)$ is called the log-live map. Note that, because of the monotonicity of the logarithm, optimizing for $\log \left(p_{\text {survive }}\right)$ is equivalent to optimizing for $p_{\text {survive }}$. We can therefore compute a $m$-step path of minimum-risk from $x_{\text {init }}$ to $x_{\text {final }}$ using (7):

$$
\min _{\substack{x_{1}, x_{2}, \ldots, x_{m}: \\ x_{1}=x_{\text {init }}, x_{m}=x_{\text {final }}}} \sum_{j=1}^{m} \log _{\text {live }}\left(x_{j}, Y\right),
$$

where the minimization is further constrained by the aircraft's kinematic model. It is also straightforward to compute paths that are Pareto-optimal with respect to the two costs: probability of survival $p_{\text {survive }}$ and path length $m$.

\section{Simulation Results}

The algorithms presented above were implemented in a simulator of military air-operations developed by the Honeywell Technology Center. Figure 2 shows a snapshot of the main simulation window displaying the battlefield during a mission that consisted of flying several aircraft from one air-base to another, passing through a region infested by enemy SAMs. In this figure, enemy radars with co-located SAMs are represented by diamonds and the friendly aircraft are represented by triangles. In this simulation, all aircraft were carrying radar detection equipment. Figure 3 shows a snapshot

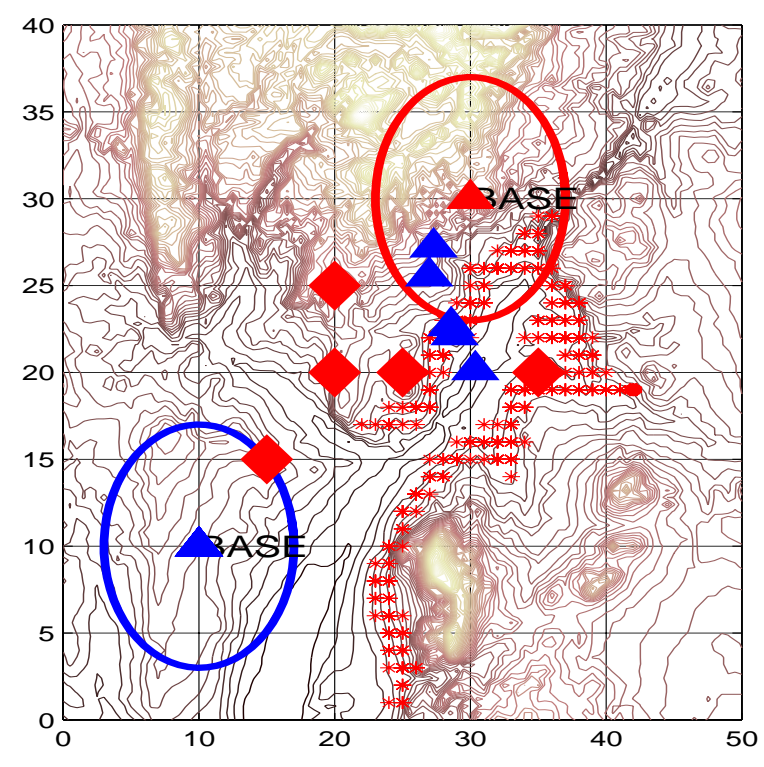

Figure 2: Battlefield

of the occupancy map defined in Section 3 (this snapshot was taken at the same time as the one in Figure 2). The probability of occupancy is encoded in the background color (lighter corresponding to a higher probability) and each " $\mathrm{x}$ " corresponds to a location where an aircraft "detected" a radar. In Figure 3, we can see that the occupancy probability is very high at four locations. These correspond to the positions of four of the radars. The fifth radar was not discovered because no aircraft flew sufficiently close to it. Note that the several false positives that can be seen in the plot were correctly "interpreted" as erroneous measurements. The log-live map defined in Section 4 is plotted in Figure 4. In this figure, darker regions indicate smaller values for the log-live map and therefore more danger, whereas lighter regions correspond to safer areas. The line superimposed in the log-live map corresponds to a path that is Pareto-optimal for the pair of costs: probability of survival and path length (with distance measured in the Manhattan sense). 


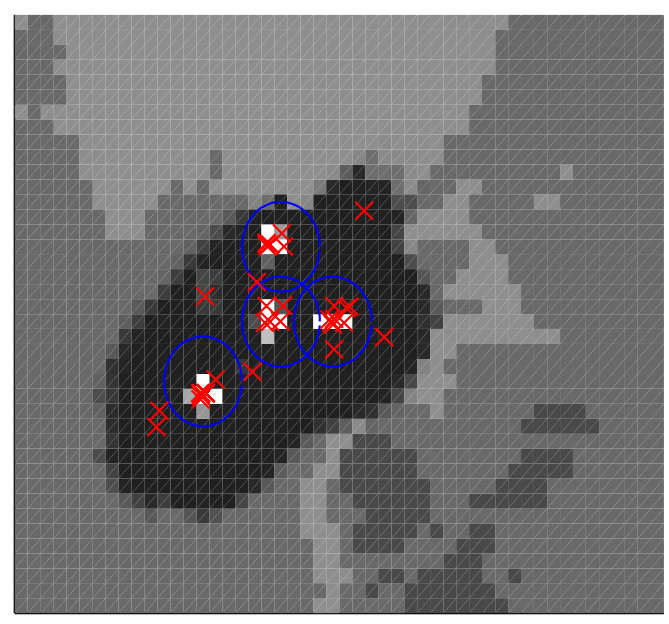

Figure 3: The occupancy map of radars

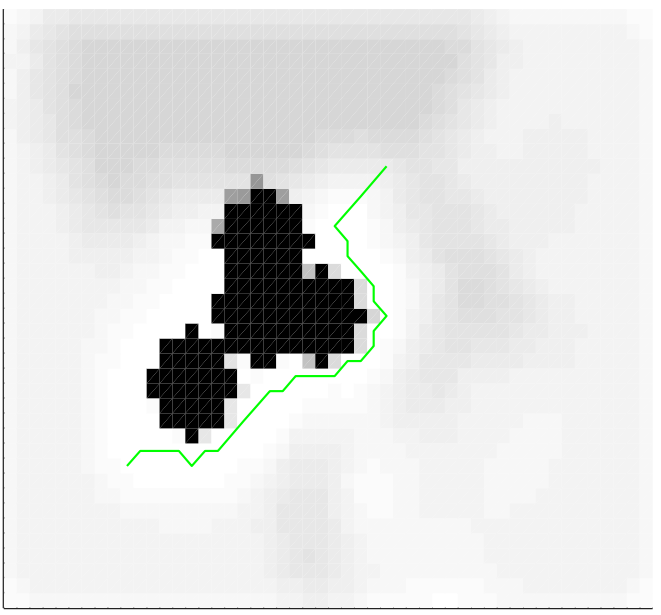

Figure 4: The log-live map of radars

\section{Conclusions}

In this paper, we developed a probabilistic formalism to fuse the information coming from aircraft sensors used to detect electromagnetic radiation originating from radars. Through the computation of several probabilistic maps, we showed how to assess the risk of missions that consist of flying an aircraft over a region infested by enemy radars and co-located SAM launchers.

We showed that, although a probabilistic map is a function defined in a high-dimensional space, it can be represented compactly by an aggregate measurement function that takes values in a space with the same dimension as the region where the radars are located. We also showed how to assess the risk of missions in airoperations and even do minimum-risk path planning without ever explicitly computing the high-dimensional probabilistic map and working simply with the aggregate measurement function.

In the formulation presented the total number of radars is assumed known. It is also assumed that they remain at fixed locations while the measurements are being collected. We are currently lifting these assumptions and considering scenarios where the radars are mobile, they can be destroyed while measurements are being taken, and new radars may enter the region.

\section{Acknowledgments}

The authors would like to acknowledge the support provided by the Honeywell Technology Center and its personnel, in particular to Datta Godbole and Jan Jelinek.

\section{References}

[1] S. P. Engelson and D. V. McDermott, "Error correction in mobile robot map learning," in Proc. IEEE Int. Conf. on Robot. and Automat., pp. 2555-2560, May 1992.

[2] F. Lu and E. Milios, "Globally consistent range scan alignment for environment mapping," $A u$ tonomous Robots, vol. 4, pp. 333-349, 1997.

[3] S. Thrun, "Learning metric-topological maps for indoor mobile robot navigation," Artificial Intelligence, vol. 99, pp. 21-71, Feb. 1998.

[4] S. Thrun, W. Burgard, and D. Fox, "A probabilistic approach to concurrent mapping and localization for mobile robots," Machine Learning and Autonomous Robots (joint issue), vol. 31, no. 5, pp. 29-53, 1998.

[5] G. Dedeoglu, M. J. Matarić, and G. S. Sukhatme, "Incremental online topological map building with a mobile robot," in Proc. of the SPIE, International Society for Optical Engineering, Sept. 1999.

[6] J. A. Castellanos, J. M. M. Montiel, J. Neira, , and J. D. Tardós, "The SPmap: a probabilistic framework for simultaneous localization and map building.," IEEE Trans. Robot. Automat., vol. 15, pp. 948-52, Oct. 1999 .

[7] B. Yamauchi and P. Langley, "Place recognition in dynamic environments," J. of Robot. Systems, vol. 14, pp. 107-120, 1997.

[8] J. P. Hespanha, H. H. Kizllocak, and Y. S. Ateşkan, "Probabilistic map building for aircrafttracking radars," tech. rep., University of Southern California, Los Angeles, CA, Sept. 2000. 